\title{
Improve Performance of Load Balancing using Artificial Bee Colony in Grid Computing
}

\author{
Deepika Nee Miku \\ M-Tech student of Department of Computer \\ Science and Application Maharshi Dayanand \\ University,,Rohtak, Haryana-124001
}

\author{
Preeti Gulia \\ Department of Computer Science and Applications \\ Maharshi Dayanand University, Rohtak, Haryana- \\ 124001
}

\begin{abstract}
Grid computing is a novel approach which solves the load balancing problems in scientific, engineering and research area. Load Balancing is a technique that can be used to improve resource utilization, to reduce MAKESPAN and to minimize number of failures. In grid environment, different algorithm for resources and data distribution is used to increase the performance and efficiency of load balancing. In grid environment Static threshold and PSO are used for load balancing. Static (fixed) threshold i.e. 3 is used for data transfer from source node to server node. Then, using PSO for data transferring that is better than, static threshold. Artificial Bee Colony Algorithm (ABC) is an optimization algorithm based on the intelligent foraging behavior of honey bee swarm. In this paper, propose a new load balancing algorithm using Artificial Bee Colony(ABC) for obtaining minimum makespan and less number of failure then, obtained results are presented and compared with static threshold and PSO.
\end{abstract}

\section{Keywords}

Grid Computing, Load Balancing, PSO, ABC

\section{INTRODUCTION}

Grid computing is the combination of computer resources from multiple administrative domains applied to achieve a goal, it is used to solve scientific, technical or business problem that requires a great number of processing cycles and needs large amounts of data. Grid computing is a distributed infrastructure which allows large scale resource sharing and system integration. Load balancing is the most important factor to improve the efficiency and performance of multiple nodes in grid based distributed environment. Workload distribution is carried out in such a way that a set of independent tasks are distributed among all the computing nodes of the grid so that the jobs are uniformly distributed and none of the nodes are overloaded or under loaded ${ }^{[1]}$. In this paper, a new load balancing algorithm is developed using $\mathrm{ABC}$ and compare the simulation results of static threshold, $\mathrm{PSO}$ and $\mathrm{ABC}$.

The rest of the paper is organized as follows. Section 2, describes related work of various swarm intelligence algorithms for load balancing. Section 3, presents importance of load balance in grid environment. Section 4, describes the concept of swarm intelligence techniques PSO and ABC algorithms. Section 5, proposes a new load balancing algorithm using $\mathrm{ABC}$. Section 6, shows the generated Experimental results in the form of table and graphical presentation of static threshold, PSO and ABC based on $M A K E S P A N$ and number of failures. Experimental results are simulated in MATLAB simulation tool. Finally, conclusion is discussed in Section 7, with suggestions for future work.

\section{RELATED WORK}

Since the last two decades, swarm intelligence (SI) has been used into many research areas because of its unique behavior which is inherent from the social insects. Swarm intelligence algorithms have been used by scientists, researchers, engineers for loads balancing of data and various optimization problems. Sowmya Suryadevevra et al., [1] proposed an Ant Colony Optimization algorithm for load balancing in grid computing is proposed which will decide the best resource to assign the jobs based on resources capacity At the same time balance the load of entire resources on grid and. The aim of this ACO is to get high throughput and thus increase the performance in grid environment. T. Kokilavani et al., [2] presents a Load balanced task scheduling which is a very important problem in complex grid environment. Author proposed Load Balanced Min-Min (LBMM) algorithm that reduces the makespan and increases the resource exploitation. The proposed method has two-phases. In the first phase, the traditional Min-Min algorithm is executed and in the second phase the tasks are rescheduled to use the unutilized resources effectively. Manish Gupta et al., [3] describes Artificial Bee Colony Algorithm (ABC) optimization algorithm based on the intelligent foraging behavior of honey bee swarm. For assigning the jobs in the system in a manner that optimized the overall performance of the application. Authors proposed, an Efficient artificial bee colony (ABC) algorithm, where they have used additional mutation and crossover operator of Genetic algorithm (GA) in the classical ABC algorithm for solving the job scheduling problem with the criterion to decrease the maximum completion time. C.Kalpana et al., [4] proposed an algorithm namely max min Particle Swarm Optimization with load balancing techniques with the comparison of Swarm Intelligence Algorithms like Ant Colony Optimization. This algorithm is based on the task scheduling in grid environment. They are calculating the QoS constraints to the PSO and ACO like, makespan, Cost and Deadline. Finally, they had balanced the load to the particle swarm optimization, and compared with all other algorithms. Lei Zhang et al., [5] adopted a heuristic approach based on Particle Swarm Optimization (PSO) algorithm to solving task scheduling problem in grid computing. Each particle is represented a possible solution and the position vector is transformed from the continuous variable to the discrete variable. The main aims to generate an optimal schedule so as to get the minimum completion time while completing the tasks. Mr. P.Mathiyalagan et al., [6] proposed an algorithm, which based on PSO for task scheduling problem on computational grids. Task scheduling algorithms based on PSO algorithm applied in computational grid environment. The aims were generating an optimal schedule so as to complete the tasks in a minimum time as well as utilizing the resources in an efficient way. T.Kokilavani et al., [7] proposed a new approach based on the argentine ant's behavior. The traditional methods try to reduce the overall response time by giving an optimized schedule. But they fail 
to produce a load balanced schedule. Moreover, they do not share the load among the available resources. Thus, the proposed method uses Ant Colony System approach to share the load among the resources for Meta Tasks. The proposed work uses one of the QoS parameter memory requirements to subdivide the task and schedule them in parallel among the available Grid resources. In this paper, PSO and ABC are popular population-based stochastic optimization algorithms are used for improve the performance of load balance in grid environment.

\section{LOAD BALANCE}

A load balancing algorithm aims to increase the utilization of resources with light load or idle resources thereby freeing the resources with heavy load. Load balancing algorithms, tries to distribute the load among all the available resources. It also aims to minimize the MAKESPAN with the effective utilization of resources. Load balancing attempts to distribute the computation load across multiple processors or machines as evenly as possible with the objective to improve performance for parallel applications.

A load balancing scheme contains three phases: information collection, decision making and data migration. In first phase, load balancer collects the information for distribution of workload and the status of computing environment and detects whether there is a load imbalance. In the second phase make the decision to calculating a best possible data distribution. In the last phase, data transfers from one overloaded node to another under loaded one ${ }^{[8]}$.

\subsection{Use of Artificial Bee Colony (ABC) For Load Balance in Grid Environment}

In grid environment, load balancing is a novel technique that is use to enhance resources, utilizing parallelism, to reduce response time through an proper distribution of the application ${ }^{[6]}$. Makespan is the total completion time taken to allocate all tasks to a resource. To minimize the makespan time and to reduce the number of failure is main objectives for load balancing. Here PSO, ABC is use for sharing load of resources so that data transferred easily on the server with minimum Makespan time, deadline constraints and less number of failures.

\section{SWARM INTELLIGENCE}

Swarm Intelligence techniques are increasingly being used for solving optimization problems. The collective intelligent behavior of insect or animal groups in nature such as flocks of birds, colonies of ants, schools of fish and swarm of bees have attracted the attention of researchers. The collective behaviors of insects or animals are called swarm behavior. Artificial intelligence deals with the collective behavior of swarms through complex interaction of individuals without supervision are referred to as swarm intelligence. Swarm intelligence has some advantages such as scalability, fault tolerance, adaptation, speed, modularity, autonomy, and parallelism. The key components of swarm intelligence are self-organization and division of labor. Bonabeau et al. have characterized four basic properties in which self-organization rely: positive feedback, negative feedback, fluctuations and multiple interactions ${ }^{[8]}$.

1. Positive feedback: means that an individual employees with other individuals by some directive, such as dancing of bees in order to lead some other bees onto a specific food source site.

2. Negative feedback: avoids all individuals accumulating on the same task by counter balancing the attraction negatively, such as abandoning the exhausted food source.

3. Fluctuations: are random behaviors of individuals in order to explore new states, such as random flights of scouts in a bee swarm.

4. Multiple interactions: are the origins of the task to be accepted out by certain rules ${ }^{[8]}$

There are using two approaches PSO and ABC for load balancing in grid computing so that resources are utilize with minimum makespan time and less number of failure.

\subsection{Particle Swarm Optimization (PSO)}

Particle swarm optimization (PSO) is a stochastic, populationbased and global optimization algorithm based on swarm intelligence, which finds a solution from optimization problem in a search space and expected social behavior.

The particle swarm simulates its behavior with social optimization. A social network is defined and assigning neighbors for each individual to interact with. Then a population of individuals defined as random guesses at the problem solutions is initialized. These individuals are candidate solutions. They are also known as the particles, hence the name particle swarm. An iterative process, to improve these candidate solutions is set in movement. The particles iteratively calculate the fitness of the candidate solutions and remember the location where they had their best achievement. The individual's best solution is called the particle best or the local best. Each particle gets this information available to their neighbors. They are also able to see their where neighbors have had success. These successes are guided to movements through the search space. Populations usually help to converging by the end of a trial, on a problem solution better than that of non-swarm approach using the same methods. Each particle represents a candidate solution to the optimization problem. The position of a particle is influenced by the best position visited by itself i.e. its own experience and the position of the best particle in its neighborhood i.e. the experience of neighboring particles. When the neighborhood of a particle is the entire swarm, the best position in the neighborhood is referred to as the global best particle, and the resulting algorithm is referred to as the gbest PSO. When smaller neighborhoods are used, the algorithm is generally referred to as the lbest PSO. The performance of each particle is measured using a fitness function that varies depending on the optimization problem. Each Particle in the swarm space represented by the following characteristics:

- The current position of the particle

- The current velocity of the particle

The particle swarm optimization which is one of the latest evolutionary optimization techniques which conducts search use a population of particles. Each particle corresponds to individual in evolutionary algorithm. Each particle by moving through the problem space has an updating position vector and updating velocity vector ${ }^{[6]}$.

1. Inertia term, forces the particle to move in the same direction as before by adjusting the old velocity. 
2. Cognitive term (Personal best), forces the particle to go back to the previous best position.

3. Social Learning term, forces the particle to move to the best previous position of its neighbors.

\subsection{Artificial Bee Colony (ABC)}

Artificial Bee Colony (ABC) algorithm is a swarm used metaheuristic algorithm. Karaboga introduced this algorithm in 2005. This algorithm simulates the foraging behavior of honey bees. This algorithm has three phases. There are employee bees, onlooker bees and scout bees. In the employee bee and the onlooker bee phases, bees build up the sources by local searchers in the neighborhood of the solutions selected based on deterministic selection in the employed bees phase and the probabilistic selection in the onlooker bees phase. In the scout bee phase, which is an analogy of abandoning exhausted food sources in the foraging process, solutions that are not beneficial anymore for search instead of them to explore new regions in the search space? The algorithm has a well-balanced exploration and development ability ${ }^{[8]}$.

These are steps of intelligent behavior of foraging bees for finding foods:

1. At the First step of the foraging process, the bees start to discover the environment randomly in order to find a food source.

2. In the second phase, after finding a food source bee becomes an employed forager and starts to exploit the discovered source. The employed bee returns to the hive with the nectar and unloads the nectar. After unloading the nectar, bees can go back to her discovered source site directly or bees can share information about her source site by performing a dance on the dance area. If her source is exhausted, she becomes a scout and starts to randomly search for a new source.

3. In the third phase, onlooker bees waiting in the hive watch the dances advertising the profitable sources and choose a source site depending on the frequency of a dance comparative to the quality of the source ${ }^{[8]}$.

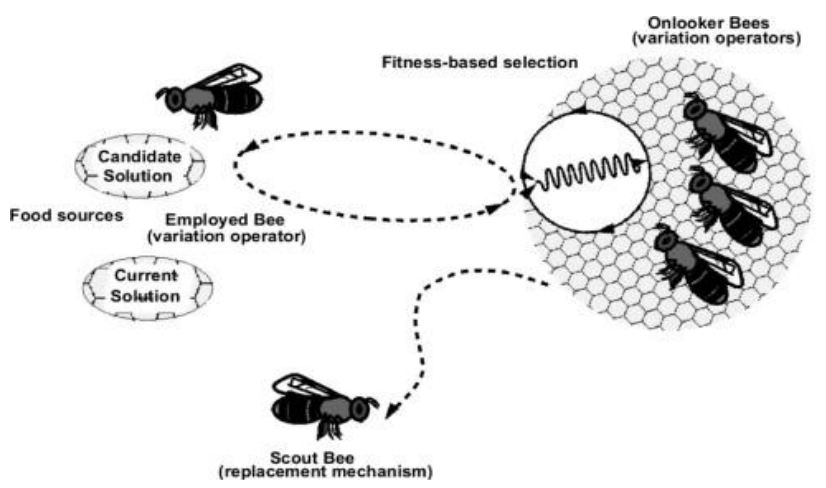

Fig 1: Bees moves for food

\section{PROPOSED WORK}

Grid Scheduling is a critical design issue of grid computing. The major objective of grid scheduling is to reduce the makespan and increase the number of tasks completed within deadline. The algorithm will be developed based on ABC to find a proper resource allocation to jobs in Grid Environment. Artificial Bee Colony (ABC) Algorithm is developed based on fixed threshold, minimum Makespan, minimum number of failure. Proposed ABC Algorithm describe as follows

\subsection{Proposed Loads balancing algorithm:}

1. Input number of nodes as input parameter to $A B C$ algorithm.

2. Generate a threshold(th) value using $A B C$ say th..

3. For $\mathrm{j}=1$ :number of rounds

4. Last $=$ store status of all nodes on grid.

5. Select any node as the server node

6. Calculate the load on each node by counting the number of requests on any node

7. For $\mathrm{i}=1$ :number of nodes

8. If load(i) $>$ th

9. Then change status(i)

10. Go to step 2 .

11. End

12. End

13. A new Request to the server node.

14. End

15. Analyze the makespan, deadline constraints.

\subsection{Threshold}

For exchanging load from a heavier loaded node to a lighter loaded node, attention must be paid not to burden the lighter node, so that it exceeds the load of the second lightest node among neighbors.

When load fairly distributing on the server node but finding a load is imbalance then to achieving this, define a THRESHOLD variable which tells how much load should exchange between nodes. Then it is calculated by subtracting LightestLoad from second LightestLoad among neighbors and the load exchange happens as long as the velocity is greater than the THRESHOLD value.

\subsection{Makespan}

MAKESPAN produced by any algorithm for a job (runs) can be calculated as follows:

MAKESPAN $=\max \left(C T\left(T_{i}, m_{j}\right)\right)$ $C T_{i j}=R_{j}+E T_{i j}$

$C T=$ Completion time of transmission nodes.

$E T_{i j}=$ Expected execution time of job $\mathrm{i}$ on resource $\mathrm{j}$

$R_{j}=$ Ready time or availability time of resource $\mathrm{j}$ after completing the previously assigned jobs.

\section{IMPLEMENTATION RESULT}

The proposed algorithm is implemented in the MATLAB and the results are shown in the table 1 and 2. The results of proposed algorithm can be compared with the results of existing technique i.e. load balancing using static threshold and the threshold using the PSO and ABC shown in table 1 and 2 respectively. The graphical comparison of the results is shown in the figure 2 and 3 . The comparison shows that the performance of purposed better than the existing algorithm.

Threshold is the value above which any resource gets overloaded. In other words, the maximum load a resource can handle is represented by the threshold value. It depends upon the number of resources available. As the number of resources are same for each run that's why threshold is same for each run i.e. 4.8750 
In $\mathrm{ABC}$ algorithm threshold is same for each run i.e. 4.9 calculating by total number nodes.

Table 1: Shows makespan time of Static threshold, PSO and $\mathrm{ABC}$

\begin{tabular}{|c|c|c|c|}
\hline Runs & $\begin{array}{c}\text { Static } \\
\text { Threshold } \\
\text { Makespan }\end{array}$ & $\begin{array}{c}\text { PSO } \\
\text { Makespan }\end{array}$ & $\begin{array}{c}\text { ABC } \\
\text { Makespan }\end{array}$ \\
\hline 1 & 523.1815 & 563.4011 & 285.0558 \\
\hline 2 & 512.6665 & 675.7394 & 288.5191 \\
\hline 3 & 628.6703 & 549.6238 & 288.3498 \\
\hline 4 & 685.5534 & 490.0471 & 283.6597 \\
\hline 5 & 679.7406 & 496.5512 & 285.2934 \\
\hline 6 & 538.6490 & 526.2188 & 283.6704 \\
\hline 7 & 492.2110 & 433.1474 & 284.8577 \\
\hline
\end{tabular}

Table 2: Shows Failure of Static threshold, PSO and ABC

\begin{tabular}{|c|c|c|c|}
\hline Runs & $\begin{array}{c}\text { Static } \\
\text { Threshold } \\
\text { Failure }\end{array}$ & $\begin{array}{c}\text { PSO } \\
\text { Failure }\end{array}$ & $\begin{array}{c}\text { ABC } \\
\text { Failure }\end{array}$ \\
\hline 1 & 9 & 7 & 7 \\
\hline 2 & 9 & 7 & 6 \\
\hline 3 & 10 & 6 & 6 \\
\hline 4 & 9 & 6 & 7 \\
\hline 5 & 9 & 6 & 7 \\
\hline 6 & 10 & 6 & 6 \\
\hline 7 & 10 & 6 & 6 \\
\hline
\end{tabular}

6.1. Graphical representation of Comparison between static threshold, PSO and ABC for number of Failure

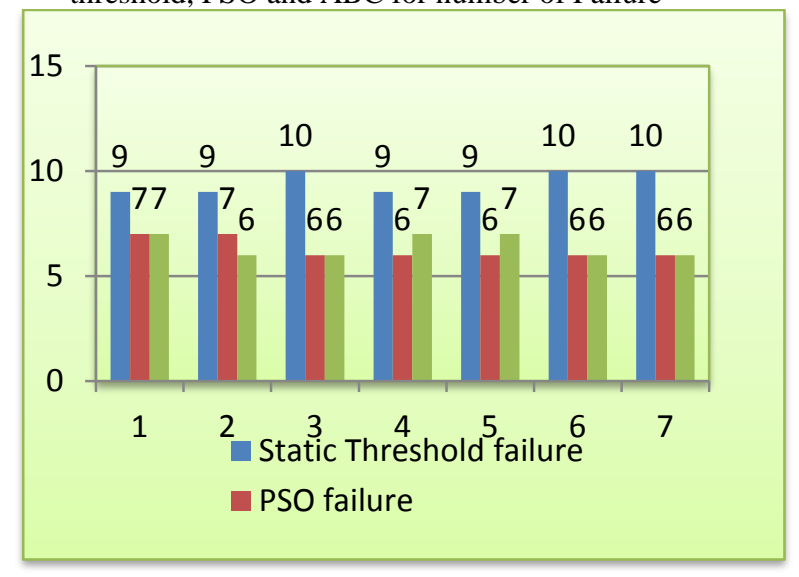

Fig 2: Number of Failures
6.2. Graph shows the Comparison and Makespan value between Static threshold, PSO and ABC.

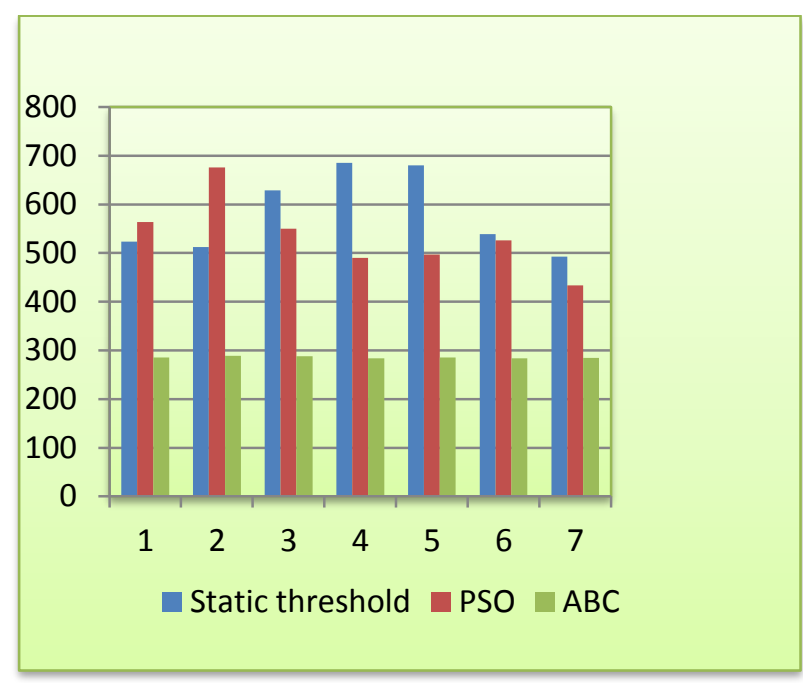

Fig 3: Makespan

\section{CONCLUSION}

Load balancing is a critical issue of grid computing. The major objective of this paper is to reduce the makespan time and minimize the number of failures. To achieve this, an algorithm is proposed for load balance based on Artificial Bee Colony optimization for proper resource allocation to jobs in grid environment. Load on resources are also balanced with the proposed load balancing algorithm. Better makespan is also achieved using newly proposed algorithm based on $\mathrm{ABC}$ due to balanced load on resources. Proposed load balancing algorithm using $\mathrm{ABC}$, gives the better results compare to static threshold and PSO. Comparison is based on minimum MAKESPAN and less number of failures. In future, these results will compare to other swarm intelligence algorithm and find out the better results.

\section{REFERENCES}

[1] Sowmya Suryadevevra, Jaishri Chourasia, Sonam Rathore, Abdul Jhummarwala, "Load Balancing in Computational Grids Using Ant Colony Optimization Algorithm", presented at International Journal of Computer \& Communication Technology (IJCCT) ISSN (ONLINE): 2231 - 0371 ISSN (PRINT): 0975 -7449 Vol-3, Iss-3, 2012.

[2] T. Kokilavani, Dr. D.I. George Amalarethinam, "Load Balanced Min-Min Algorithm for Static Meta-Task Scheduling in Grid Computing", International Journal of Computer Applications (0975 - 8887) Volume 20- No.2, April 2011

[3] Manish Gupta, Govind Sharma, “ An Efficient Modified Artificial Bee Colony Algorithm for Job Scheduling Problem" ,International Journal of Soft Computing and Engineering (IJSCE) ISSN: 2231-2307, Volume-1, Issue6, January 2012

[4] C.Kalpana, U.Karthick Kumar, R.Gogulan, "Max-Min Particle Swarm Optimization Algorithms with Load Balancing for Distributed Task Scheduling on the Grid Environment", IJCSI International Journal of Computer Science Issues, Vol. 9, Issue 3, No 1, May 2012. 
[5] Lei Zhang, Yuehui Chen, Runyuan Sun, Shan Jing and Bo Yang, "A Task Scheduling Algorithm Based on PSO for Grid Computing" , International Journal of Computational Intelligence Research. ISSN 0973-1873 Vol.4, No.1 , pp. 37-43,2008.

[6] Mr. P.Mathiyalagan, U.R.Dhepthie, Dr. S.N.Sivanandam,"Grid Scheduling using Enhanced PSO Algorithm", P.Mathiyalagan et al. / (IJCSE) International Journal on Computer Science and Engineering Vol. 02, No. 02, 2010, 140-145.
[7] T.Kokilavani, Dr. D.I. George Amalarethinam, "Memory Constrained Ant Colony System For Task Scheduling In Grid Computing", International Journal of Grid Computing \& Applications (IJGCA) Vol.3, No.3, September 2012.

[8] Bahriye Akay, Dervis Karaboga," A modified Artificial Bee Colony algorithm for real-parameter optimization", Inform. Sci. (2010), doi:10.1016/j.ins.2010.07.015. Elsevier Inc. 